\title{
Constraining the equation of state of nuclear matter with gravitational wave observations: Tidal deformability and tidal disruption
}

\author{
Andrea Maselli, ${ }^{1}$ Leonardo Gualtieri, ${ }^{1}$ and Valeria Ferrari ${ }^{1}$ \\ ${ }^{1}$ Dipartimento di Fisica, Università di Roma "La Sapienza" ES Sezione, \\ INFN Roma1, P.A. Moro 5, 00185, Roma, Italy.
}

(Dated: June 16, 2021)

\begin{abstract}
We study how to extract information on the neutron star equation of state from the gravitational wave signal emitted during the coalescence of a binary system composed by two neutron stars or a neutron star and a black hole. We use Post-Newtonian templates which include the tidal deformability parameter and, when tidal disruption occurs before merger, a frequency cut-off. Assuming that this signal is detected by Advanced LIGO/Virgo or ET, we evaluate the uncertainties on these parameters using different data analysis strategies based on the Fisher matrix approach, and on recently obtained analytical fits of the relevant quantities. We find that the tidal deformability is more effective than the stellar compactness to discriminate among different possible equations of state.

PACS numbers: 04.30.Tv,97.60.Jd, 97.60.Lf, 04.25.dk,
\end{abstract}

\section{INTRODUCTION}

Black hole (BH)-neutron star (NS) mergers are promising sources of gravitational waves (GWs) to be detected by interferometric detectors of second (AdvLIGO/Virgo [1]) and third (ET, 2]) generation. The detection of GW signals from these processes will provide valuable information on the NS internal structure, which would be impossible to obtain otherwise. For this reason, in recent years much effort has been devoted to model the signal emitted during the latest phases of the inspiralling of NSNS and NS-BH binaries, when the imprint of the equation of state $(\mathrm{EoS})$ on the signal is more pronounced (see e.g. [3-13]). However, the best strategy to extract as much of information as possible on the NS EoS from a detected signal is still under debate. Addressing this question is the main scope of this work. We shall focus on the latest inspiralling, when NS deformations can be large, and we shall use two quantities which encode information on the NS EoS, to compare different strategies: the tidal deformability $\lambda$ (which will be defined in Section II) and the stellar compactness $\mathcal{C}=M_{\mathrm{NS}} / R_{\mathrm{NS}}$, where $M_{\mathrm{NS}}$ is the NS gravitational mass and $R_{\mathrm{NS}}$ its radius.

If the companion is a black hole, under appropriate conditions a NS can be disrupted by the tidal interaction before being swallowed. In this case the GW-signal exhibits a frequency cut-off $f_{\text {cut }}$ which also depends on the EoS. Most of the articles cited above include either $\lambda$ (or $\mathcal{C})$ or $f_{\text {cut }}$ in the model; in this paper, we shall include both parameters in the signal template and, using recently proposed analytic fits of $\mathcal{C}(\lambda)\left[14\right.$ and $f_{\text {cut }}(\mathcal{C})$ [8], and a Fisher matrix approach, we shall evaluate whether a joint analysis can improve the possibility to gain information on the NS EoS with respect to an approach which includes only one of these quantities. It should be stressed that tidal disruption in BH-NS or NS-NS binaries is of particular interest, since they are have been invoked as possible engines of Short Gamma-Ray Bursts [15] (for a review on the subject, see e.g. [16]).
In addition, we shall discuss which is the most useful quantity to be used in order to constrain the EoS of matter in the NS interior. In the literature, it is often assumed that the most valuable information that GW physicists (or astrophysicists) can provide to nuclear physicists is the value of the NS radius $R_{\mathrm{NS}}$ or, equivalently, the star compactness $\mathcal{C}$ 17. A possibility is to estimate $\lambda$ from a detected GW signal and then derive $R_{\mathrm{NS}}$ from it [14, 18. On the other hand, $\lambda$ itself could be used to constrain the NS EoS [7, 12. We shall compare these two approaches, assessing their ability to discriminate among different EoS.

In this paper we shall consider the advanced GW detectors AdvLIGO/Virgo, and third generation detectors like ET. Since ET is expected to detect GW signals from coalescing binaries up to $2 \mathrm{Gpc}$, the cosmological redshift will be consistently included in the template waveforms.

The plan of the paper is the following. In Section II we briefly introduce the tidal deformability $\lambda$ and the cut-off frequency and in Section III we show how these parameters can be included in a waveform template. In Section IV we discuss how $\lambda, f_{\text {cut }}$ and $\mathcal{C}$ can be used to extract information on the NS EoS. In Section $\nabla$ we discuss whether the tidal deformability has to be preferred to the stellar compactness as a parameter to discriminate among different EoS. In Section VI we draw our conclusions. Finally, in Appendix A we sketch the basic elements of parameter estimation theory. We remark that Section IV] refers to BH-NS binary systems, while in the rest of the paper we consider both BH-NS and NS-NS binaries.

\section{MODELING TIDAL INTERACTIONS IN BH-NS AND NS-NS BINARIES}

In this Section we briefly introduce the parameters which depend on the equation of state of matter in the NS interior, and which appear in the late inspiralling GW 
signal: the Love number, describing the star deformability, and the cut-off frequency which appears in the emitted signal when a NS is tidally disrupted by a companion $\mathrm{BH}$ before merging.

\section{A. The Love number}

NS tidal deformations can be described in terms of a set of parameters, the Love numbers [3, 4, which relate the mass multipole moments of the deformed star with the external tidal multipole moments. The quantity which encodes most of the information on stellar deformation is the quadrupole Love number $k_{2}$, given by

$$
Q_{i j}=-\frac{2}{3} k_{2} R_{\mathrm{NS}}^{5} C_{i j}
$$

where $Q_{i j}$ is the star traceless quadrupole tensor, and $C_{i j}=e_{(0)}^{\alpha} e_{(i)}^{\beta} e_{(0)}^{\gamma} e_{(j)}^{\delta} R_{\alpha \beta \gamma \delta}$ is the tidal tensor of the gravitational field $\left(e_{(\mu)}^{\alpha}\right.$ is the parallel transported tetrad attached to the deformed star, and $R_{\alpha \beta \gamma \delta}$ is the Riemann tensor). The ratio between the quadrupole and the tidal tensors,

$$
\lambda=\frac{2}{3} k_{2} R_{\mathrm{NS}}^{5},
$$

is the NS tidal deformability.

$k_{2}$ and $\lambda$ can be computed by studying the quadrupolar, stationary perturbations induced by a test tidal field acting on the NS [3, 19, 20. It has recently been shown [18 that the Love number - or, more precisely, the tidal deformability - is related to the momentum of inertia of the star and to its rotation-induced quadrupole moment by the so-called "I-Love-Q relations", which are almost independent of the EoS and of the NS mass; a similar universal relation can be found between the tidal deformability and the NS compactness $\mathcal{C}$ [14].

Tidal interactions affect the gravitational signal emitted by coalescing compact binaries, and these effects are currently included in the gravitational waveforms within approximation schemes, as the Post-Newtonian expansion (PN), or the Effective One Body (EOB) formalism. In these frameworks it has been shown [21, 22, that the leading contribution is given by an extra term in the phase of the signal, which is proportional to the tidal deformability. Therefore, the detection of a signal emitted in a NS-BH or a NS-NS coalescence can, in principle, allow to determine $\lambda$ or $k_{2}$. The accuracy with which second and third generation detectors will be able to measure $\lambda$ has recently been estimated through a data analysis based on Fisher matrix or Markov Chain Monte Carlo techniques (see App. A and 23] for a general discussion on the Fisher matrix formalism; see [24] for the Markov Chain Monte Carlo approach). Using the EOB formalism and a large class of EoS, it has been shown that $\lambda$ can be measured by advanced detectors if the signal emitted by a coalescing NS-NS binary is detected with signal-to-noise ratio (SNR) $\rho=16$ [12. In the case of BH-NS binaries, a similar estimate has been carried out using phenomenological waveforms obtained matching PN templates with the outcome of fully relativistic simulations, finding that the tidal deformability can be extracted for sources whose mass ratio $q=M_{\mathrm{BH}} / M_{\mathrm{NS}}$ (where $M_{\mathrm{BH}}$ is the $\mathrm{BH}$ mass) is $q=2,3$, at $d=100 \mathrm{Mpc}$ with 10\%-40\% accuracy by AdvLIGO/Virgo, and with an order of magnitude better accuracy by the Einstein Telescope [11. Markov Chain Monte Carlo approaches have been employed in 25, showing that, for NS-NS binaries, few tens of detections by advanced interferometers will be required to strongly constrain $\lambda$.

\section{B. The frequency cut-off}

In the coalescence of a BH-NS binary system, the NS can be tidally disrupted before plunging into the $\mathrm{BH}$. This phenomenon can occur if the star is very deformable, if the $\mathrm{BH}$ is rapidly spinning, or if the mass-ratio is very small (see e.g. [8, 10, 26, 27]). Otherwise, the NS behaves nearly as a point particle until the very last stages of the coalescence, and it is swallowed by the $\mathrm{BH}$ without being disrupted.

When the NS is tidally disrupted, the gravitational signal exhibits a clear signature: the waveform amplitude steeply drops down at a cut-off frequency $f_{\text {cut }}$ [5] (see also [16] for a general description of the process). So far, studies of $f_{\text {cut }}$ for BH-NS binaries have been carried out with either semi-analytical approaches [5, 6] or fully numerical simulations [8, 26, 27]. The first proposal of using a measure of $f_{\text {cut }}$ to determine $R_{\mathrm{NS}}$ goes back to more than a decade ago [5]; in that article a relation, based on simplifying assumptions and derived in a Newtonian framework, was proposed to connect $f_{\text {cut }}$ with $R_{\mathrm{NS}}$. A much more accurate relation between $f_{\text {cut }}$ and the compactness $\mathcal{C}$ (and then $R_{\mathrm{NS}}$ ) has recently been proposed [8, based on the results of fully relativistic simulations of BH-NS coalescences:

$$
\ln \left(f_{\text {cut }} m\right)=(3.87 \pm 0.12) \ln \mathcal{C}+(4.03 \pm 0.22)
$$

where $m \equiv M_{\mathrm{NS}}+M_{\mathrm{BH}}$. These simulations have been performed assuming that the $\mathrm{BH}$ is non-rotating, and that the mass-ratio of the binary is $q=2$, therefore the fit (3) can only be trusted under these assumptions. In the next two Sections we shall exploit the fit (3); therefore, we shall focus on BH-NS binaries with zero BH spin and $q=2$.

Realistic BH-NS binary systems are thought to have larger values of the mass ratio and a large $\mathrm{BH}$ rotation rate 28 . The analysis carried out in the present paper can easily be extended to such configurations, once fully relativistic simulations of these systems will be available. 


\section{THE GRAVITATIONAL WAVE TEMPLATES}

Post-Newtonian GW templates which include finite size effects, accurately describe the evolution and the gravitational emission of BH-NS and NS-NS binaries up to the last orbits before the merger 29 33 . However, they do not encode information about NS tidal disruption which may possible occur. This can be done, as a first approximation, including in the gravitational template a cut-off at the frequency $f_{\text {cut }}$; this value will be treated as one of the parameters of the template. We shall model the inspiral waveform as follows:

$$
\bar{h}_{\mathrm{PN}}(f)= \begin{cases}h_{3 \mathrm{PN}} & f<f_{\text {cut }} \\ h_{3 \mathrm{PN}} \times \Theta\left(f, f_{\text {cut }}\right) & f_{\text {cut }} \leq f \leq 2 f_{\text {cut }} \\ 0 & f>2 f_{\text {cut }}\end{cases}
$$

where

$$
h_{3 \mathrm{PN}}(f)=\mathcal{A}_{3 \mathrm{PN}}(f) e^{i\left(\psi_{\mathrm{PP}}+\psi_{\mathrm{T}}\right)}
$$

is the standard TaylorF2 approximant of the GW signal in the frequency domain [34. In this work we consider optimally oriented observers, such that the 3 PN amplitude reads:

$$
\begin{aligned}
\mathcal{A}_{3 \mathrm{PN}}(f) & =\mathcal{A} f^{-7 / 6} \sum_{k=0}^{6} \beta_{k}(m \pi f)^{k / 3}, \\
& =\sqrt{\frac{5}{24}} \frac{\mathcal{M}^{5 / 6}}{\pi^{2 / 3} d} f^{-7 / 6} \sum_{k=0}^{6} \beta_{k}(m \pi f)^{k / 3},
\end{aligned}
$$

where $\mathcal{M}=m \nu^{3 / 5}$ is the chirp mass, $m=M_{\mathrm{BH}}+M_{\mathrm{NS}}$ and $\nu=M_{\mathrm{BH}} M_{\mathrm{NS}} / m^{2}$, and $d$ is the source distance. The coefficients $\beta_{k}$ are given by Eq. (42) of 35$]^{1}$. Finally, $\psi_{\mathrm{PP}}$ represents the point-particle contribution to the GW phase, currently known at the $3.5 \mathrm{PN}$ order [36, while $\psi_{\mathrm{T}}$ describes the effects of tidal interactions [21, 22, and it is given by

$$
\begin{aligned}
\psi_{\mathrm{T}}= & -\frac{117}{8 \nu} \frac{\tilde{\lambda}}{m^{5}} x^{5 / 2}\left(1+2.5 x-\pi x^{3 / 2}\right. \\
& \left.+8.51 x^{2}-3.92 \pi x^{5 / 2}\right)
\end{aligned}
$$

where $x=(m \pi f)^{2 / 3}$; the rescaled tidal deformability $\tilde{\lambda}$ is related to the tidal deformability $\lambda$ (defined in Eq. (2)) by

$$
\tilde{\lambda}=\frac{1+12 q}{26} \lambda
$$

\footnotetext{
1 We note that $\mathcal{A}_{3 \mathrm{PN}}$ contains some imaginary contributions; hereafter, when referring to the GW spectrum $f h(f)$, we shall mean the modulus $|f h(f)|$.
}

in the case of BH-NS binaries, and by

$$
\tilde{\lambda}=\frac{1}{26}\left[(1+12 q) \lambda_{\mathrm{NS}_{1}}+\frac{q+12}{q} \lambda_{\mathrm{NS}_{2}}\right],
$$

for NS-NS binaries (note that in this case, if $q=1 \tilde{\lambda}=\lambda$ ). The function $\Theta\left(f, f_{\text {cut }}\right)$ appearing in the extended template (4) reproduces the sharp decrease in the amplitude corresponding to tidal disruption; we choose it to have the form

$$
\Theta\left(f, f_{\text {cut }}\right)=e^{-\alpha\left(f / f_{\text {cut }}-1\right)} .
$$

We have also considered different forms of the cut-off function $\Theta$, finding that such change does not significantly affect our results. To compare the approximate waveform given in (4) with the waveforms produced by numerical simulations of binary coalescence, $h_{\mathrm{NR}}$, it is useful to compute the overlap of the two signals, given by

$$
\mathcal{O}\left(\bar{h}_{\mathrm{PN}}, h_{\mathrm{NR}}\right)=\frac{\left(\bar{h}_{\mathrm{PN}} \mid h_{\mathrm{NR}}\right)}{\sqrt{\left(\bar{h}_{\mathrm{PN}} \mid \bar{h}_{\mathrm{PN}}\right)} \sqrt{\left(\bar{h}_{\mathrm{NR}} \mid \bar{h}_{\mathrm{NR}}\right)}},
$$

where (see Appendix A)

$$
(g \mid h)=2 \int \frac{\tilde{h}(f) \tilde{g}^{\star}(f)+\tilde{h}^{\star}(f) \tilde{g}(f)}{S_{h}(f)} d f,
$$

$S_{h}(f)$ is the detector noise spectral density, and the integration is performed in the frequency range $\left[f_{\text {cut }}, 2 f_{\text {cut }}\right]$. It is worth remarking that although the gravitational waveform given by Eq. (4) represents only a coarse approximation of the true signal around the tidal disruption frequency, it is accurate enough for the study we intend to carry on in this work.

For binary sources at cosmological distances, which are the main target of the third generation detector ET, the GW signal has to be properly redshifted. As discussed in 23, 37, the point particle phase $\psi_{\mathrm{PP}}$ and the signal amplitude $\mathcal{A}_{3 \mathrm{PN}}$ are invariant under the general transformation $(f, \mathcal{M}, d, t) \rightarrow(f / \xi, \mathcal{M} \xi, d \xi, t \xi)$, where $\xi=1+z$. This means that using the point-particle approximation we can only set constraints on the redshifted chirp-mass $\mathcal{M}_{z}=\mathcal{M}(1+z)$ and on the luminosity distance $d_{\mathrm{L}}=d(1+z)$ : if the proper distance $d$ is unknown, it is impossible to disentangle the mass parameter and the redshift. However, the tidal phase $\psi_{\mathrm{T}}$ depends on the unredshifted, rest-frame mass components $M_{\mathrm{BH}}, M_{\mathrm{NS}}$, and then allows to break this degeneracy.

In this work we consider binary systems up to $z \simeq 0.5$. The redshift $z$ is given in terms of the distance $d$ by the relation

$$
d=\frac{1}{H} \int_{0}^{z} \frac{d z^{\prime}}{\sqrt{\Omega_{m}\left(1+z^{\prime}\right)^{3}+\Omega_{\Lambda}}},
$$

where $H=68 \mathrm{~km} / \mathrm{Mpc} / \mathrm{s}$ is the Hubble constant, $\Omega_{m}=$ 0.317 is the total matter density, and $\Omega_{\Lambda}=0.683$ is the 
dark energy density measured by the Planck satellite [38]. In order to include the cosmological redshift in the waveform, we (i) replace the quantities $(f, m, d)$ with the redshifted quantities $\left(\bar{f}, \bar{m}, d_{\mathrm{L}}\right)$ rescaled with $\xi=1+z$ as discussed above (this does not change the functional form of the waveform); (ii) modify the form of the tidal contribution and of the cut-off, Eqns. (7) and (10), as follows:

$$
\begin{aligned}
\psi_{\mathrm{T}}(\bar{f})= & -\frac{117}{8 \nu} \frac{(1+z)^{5} \tilde{\lambda}}{\bar{m}^{5}} x^{5 / 2}\left(1+2.5 x-\pi x^{3 / 2}\right. \\
& \left.+8.51 x^{2}-3.92 \pi x^{5 / 2}\right) \\
\Theta\left(\bar{f}, f_{\text {cut }}\right)= & e^{-\alpha\left(\bar{f}(1+z) / f_{\text {cut }}-1\right)} .
\end{aligned}
$$

Once the GW signal from a NS-NS or NS-BH inspiral has been detected, it will be possible to extract the values of the binary parameters comparing the data with the GW template, through a matched filtering technique. Assuming Gaussian noise and sufficiently high signal-to-noise ratio, the parameter variance can be estimated using the Fisher matrix approach (see for example [4, 17, 12, 23, 39, and Appendix A of this paper), which we here discuss.

In the next Section we shall restrict to the case of GWsignals emitted by a NS-BH coalescing binary which exhibits the feature associated to NS disruption, i.e. $f_{\text {cut }}$. In this case, the GW template defined in Eq. (4) depends on the set of parameters

$$
\boldsymbol{\theta}=\left(\ln \mathcal{A}, t_{c}, \phi_{c}, \ln \mathcal{M}, \ln \nu, \tilde{\lambda}, f_{\text {cut }}\right),
$$

where $t_{c}$ and $\phi_{c}$ are the time and the phase at the coalescence. We do not include the cosmological redshift $z$ among the set of parameters 16 , since we assume that $z$ is known a priori by coincidence measurements in the electromagnetic band. Therefore, our computation will eventually yield the uncertainties on the un-redshifted parameters.

The parametrization (16) leads to a $7 \times 7$ covariance matrix (see Appendix A), whose diagonal elements represent the standard deviation or the parameters 16 . We remark that $\ln \mathcal{A}$ is uncorrelated with the other parameters 39]; therefore, we will restrict our analysis to the remaining 6 parameters. We also remark that once $\tilde{\lambda}$, $\mathcal{M}$ and $\nu$ are estimated, the tidal deformability $\lambda$ can be computed from Eq. (8) or (9).

Since, as we shall show in Sec. II] the cut-off frequency $f_{\text {cut }}$ will mostly be accessible to third generation interferometers, in the following analysis we will use the noise spectral density of the Einstein Telescope described by the fit

$$
\begin{aligned}
\frac{S_{h}}{S_{0}}=x^{-4.1} & +186 x^{-0.69}+233\left[1+31 x-65 x^{2}+52 x^{3}+\right. \\
& \left.-42 x^{4}+10 x^{5}+12 x^{6}\right] /\left[1+14 x-37 x^{2}+\right. \\
& \left.+19 x^{3}+27 x^{4}\right]
\end{aligned}
$$

with $\bar{f} \geq f_{s}=10 \mathrm{~Hz}, S_{0}=10^{-52} \mathrm{~Hz}^{-1}$ and $x=\bar{f} / f_{0}$, being $f_{0}=200 \mathrm{~Hz}$ a scaling frequency 40.

\section{DATA-ANALYSIS STRATEGIES}

In this Section we discuss how the cut-off frequency $f_{\text {cut }}$, identified in a detected gravitational wave signal emitted by a BH-NS binary, can be used to extract information on the NS EoS. As discussed in Section III, the gravitational waveform depends on two quantities which carry the imprint of the NS EoS: the deformability $\lambda$ and - if tidal disruption occurs - the cut-off frequency $f_{\text {cut }}$. These quantities can both be related to the NS compactness $\mathcal{C}$, using the $f_{\text {cut }}(\mathcal{C})$ fit $(3)$ found in [8, and the $\lambda(\mathcal{C})$ fit found in [14:

$\mathcal{C}=0.371-0.0391 \ln \left[\frac{\lambda}{M_{\mathrm{NS}}^{5}}\right]+0.001056\left[\ln \left(\frac{\lambda}{M_{\mathrm{NS}}^{5}}\right)\right]^{2}$,

where $\lambda(\tilde{\lambda})$ is given by Eq. (8). We remind that Eq. 18 is found to reproduce the values of the star compactness with an accuracy greater $3 \%$, for a large class of EoS. In the following, we shall denote by $\mathcal{C}_{\lambda}$ the NS compactness obtained from the fit (18), and by $\mathcal{C}_{\text {cut }}$ the NS compactness obtained from the fit (3).

We propose and compare two data-analysis strategies to extract information on the neutron star equation of state, which employ these two fits in a different way.

With the first strategy, we assume that the unknown parameters are

$$
\boldsymbol{\theta}=\left(t_{c}, \phi_{c}, \ln \mathcal{M}, \ln \nu, \tilde{\lambda}, f_{\text {cut }}\right),
$$

and estimate the corresponding errors $\sigma_{i}, i=1,6$, with a $6 \times 6$ covariance matrix. We then write the relation (3) between $f_{\text {cut }}$ and the NS compactness, in the form

$$
\ln \left(f_{\text {cut }} m\right)=\left(a \pm \sigma_{a}\right) \ln \mathcal{C}_{\text {cut }}+\left(b \pm \sigma_{b}\right),
$$

where $a, \sigma_{a}, b, \sigma_{b}$ are given in eq. (3). Assuming the errors to be uncorrelated, we compute the variance on $\mathcal{C}_{\text {cut }}$ as:

$$
\sigma_{\mathcal{C}_{\text {cut }}}^{2}=\sum_{p_{i}}\left(\frac{\partial \mathcal{C}_{\text {cut }}}{\partial p_{i}}\right)^{2} \sigma_{p_{i}}^{2}
$$

where $p_{i}=\left\{a, b, m, f_{\text {cut }}\right\}$ Eq. 21 provides the error on the NS compactness derived from the frequency cutoff only.

Using the fit 18 we then compute the error on the NS compactness directly from the tidal deformability $\lambda$, as

$$
\sigma_{\mathcal{C}_{\lambda}}^{2}=\sigma_{\text {fit }}^{2}+\left(\frac{\partial \mathcal{C}_{\lambda}}{\partial \ln \tilde{\lambda}}\right)^{2} \sigma_{\ln \tilde{\lambda}}^{2}+\left(\frac{\partial \mathcal{C}_{\lambda}}{\partial M_{\mathrm{NS}}}\right)^{2} \sigma_{M_{\mathrm{NS}}}^{2}
$$

2 The error on the total mass $m$ is easily computed from the chirp mass $\mathcal{M}$ and the symmetric mass ratio $\nu$ as

$\sigma_{m}^{2}=\left(\frac{\partial m}{\partial \mathcal{M}}\right)^{2} \sigma_{\mathcal{M}}^{2}+\left(\frac{\partial m}{\partial \nu}\right)^{2} \sigma_{\nu}^{2}+2 \frac{\partial m}{\partial \mathcal{M}} \frac{\partial m}{\partial \nu} \operatorname{cov}(\mathcal{M}, \nu)$

where $\operatorname{cov}(\mathcal{M}, \nu)$ is the $\mathcal{M}-\nu$ element of the covariant matrix for the parameters 19 . 
where we set $\sigma_{\text {fit }}=0.03 \mathcal{C}$. The latter has been estimated in 14 and it corresponds to the largest relative discrepancy between the value of $\lambda$ obtained from the fit and the value computed solving the equations of stellar perturbations, for a set of EoS covering a large range of stiffness. In addition, since the two masses are comparable (in this paper we only consider low values of the mass ratio) and the total error is dominated by $\sigma_{\text {fit }}$, we assume $\sigma_{M_{\mathrm{NS}}}$ to be of the same order of $\sigma_{m}$. The value of the NS compactness $\mathcal{C}$ is obtained combining the information given by the two fits (21) and (18), and is the weighted mean

$$
\mathcal{C}=\frac{\mathcal{C}_{\text {cut }} / \sigma_{\mathcal{C}_{\text {cut }}}^{2}+\mathcal{C}_{\lambda} / \sigma_{\mathcal{C}_{\lambda}}^{2}}{\sigma_{\mathcal{C}_{\text {cut }}}^{-2}+\sigma_{\mathcal{C}_{\lambda}}^{-2}}
$$

with variance

$$
\sigma_{\mathcal{C}}^{2}=\frac{1}{\sigma_{\mathcal{C}_{\text {cut }}}^{-2}+\sigma_{\mathcal{C}_{\lambda}}^{-2}}
$$

The second strategy consists in expressing the information on the neutron star internal composition in terms of one single parameter, the rescaled tidal deformability $\tilde{\lambda}$. We combine the fits (3) and (18) (using Eq. (8)) to express $f_{\text {cut }}$ in terms of $\lambda$, and substitute this expression in the waveform template given by eq. (4). We then compute the $5 \times 5$ covariance matrix, for the set of variables $\boldsymbol{\theta}=\left(t_{c}, \phi_{c}, \ln \mathcal{M}, \ln \nu, \tilde{\lambda}\right)$. The value of the NS compactness $\mathcal{C}$ and its variance are then obtained from the fit (18) and Eq. 23]. This procedure reduces the data-analysis computational cost with respect to the previous strategy, since it does not include the parameter $f_{\text {cut }}$.

\section{A. The binary models}

In order to be fully consistent with the fit (3) derived in [8], in this Section we shall consider the same binary models used in [8, i.e. BH-NS binaries with mass ratio $q=2$, with the neutron star modeled using a set of piecewise polytropes [41]. In particular, the core is described by a polytropic $p=K \rho^{\Gamma}$ with $\Gamma=3$, and such that $p$ is equal to a given value $p_{1}$ at density $\rho_{1}=5.0119 \times 10^{14} \mathrm{~g} \mathrm{~cm}^{-3}$; the crust is described by a polytropic with $\Gamma=1.3569$, such that $p=1.5689 \times 10^{31}$ dyn $\mathrm{cm}^{-2}$ at density $\rho=10^{13} \mathrm{~g}$ $\mathrm{cm}^{-3}$. The overall EoS depends on the value of the parameter $p_{1}$. In [8] four EoS have been considered, corresponding to four values of $p_{1}$, and named (from the stiffest to the softest) $2 \mathrm{H}, \mathrm{H}, \mathrm{HB}$ and $\mathrm{B}$.

In Fig. 1 we show the gravitational wave spectra $f h(f)$ extracted from figure 9 of $[8$; the spectra refer to $\mathrm{BH}-$ NS systems with mass ratio $q=2$ and $M_{\mathrm{NS}}=1.2 M_{\odot}$ located at the distance $d=100 \mathrm{Mpc}$; in addition we plot the noise spectral densities of Advanced LIGO [1] and of the Einstein Telescope (ET) 2, the values of the cutoff frequency (empty circles) derived from Eq. (3), and those of the Innermost Circular Orbit (ICO - grey circles), computed by minimizing the total Post-Newtonian binding energy of the two body system as in [36, 42].
From Fig. 1 we can immediately draw the following considerations: (i) the NS is tidally disrupted before the ICO only for the stiffest EoS, 2H; (ii) for the considered systems, third generation detectors like ET are needed to unambiguously detect $f_{\text {cut }}$. We have also considered binaries with $M_{\mathrm{NS}}=1.35 M_{\odot}$, finding that the same conclusions apply. Moreover, increasing the neutron star mass, the compactness increases, leading to higher cutoff frequencies.

Therefore, in applying the proposed data analysis strategies we shall only consider NSs modeled by the 2H EoS (defined by $p_{1}=10^{13.95} \mathrm{~g} / \mathrm{cm}^{3}$ ), with $M_{\mathrm{NS}}=$ $1.20 M_{\odot}, R_{\mathrm{NS}}=15.10 \mathrm{~km}$ and $M_{\mathrm{NS}}=1.35 M_{\odot}, R_{\mathrm{NS}}=$ $15.20 \mathrm{~km}$. From the fit (3) we find $f_{\text {cut }}=796.67 \mathrm{~Hz}$ and $f_{\text {cut }}=1085.51 \mathrm{~Hz}$, respectively. Using the approach of [3] we have computed the Love number for these configurations, finding $k_{2}=0.1452$ and $k_{2}=0.1342$, respectively. As in [8, we have considered non-spinning BHs with mass ratio $q=M_{\mathrm{BH}} / M_{\mathrm{NS}}=2$. As discussed in Section IIB, the analysis of this work can easily be extended to more general configurations, once fully relativistic simulations will provide a fit analogous to Eq. (3).



FIG. 1. Gravitational wave spectra $f h(f)$ extracted from 8 , for $q=2$ and $M_{\mathrm{NS}}=1.2 M_{\odot}$ at a distance $d=100 \mathrm{Mpc}$. Empty and grey circles refer to the cut-off and the ICO frequencies. We also show the noise spectral density of Advanced LIGO and the Einstein Telescope.

The coefficient $\alpha$ in Eq. 10 has been chosen to minimize the discrepancy between the numerical spectra given in 8 and the analytical spectra given by eq. (4). This value is $\alpha=1.55$. As a further check, we have compared the waveform given in (4) with those obtained from the numerical simulations of 8 , computing the overlap integrals 111 in the frequency range $\left[f_{\text {cut }}, f_{\text {end }}\right]$ (where $f_{\text {end }}$ is the highest value of frequency in the numerical data). We find $\mathcal{O}\left(\bar{h}_{\mathrm{PN}}, h_{\mathrm{NR}}\right)=0.9991$ for $M_{\mathrm{NS}}=1.2 M_{\odot}$ and $\mathcal{O}\left(\bar{h}_{\mathrm{PN}}, h_{\mathrm{NR}}\right)=0.9997$ for $M_{\mathrm{NS}}=1.35 M_{\odot}$. These values are compatible with the standard accuracy threshold used for GW detection, i.e. $1-\mathcal{O}<0.005$ [3] (assuming that the same threshold will hold for the ET data analysis). We note that, if we compute the overlap between the waveform containing only the inspiral part of the signal $h_{3 \mathrm{PN}}$, and the numerical one, we obtain 
$\mathcal{O}\left(h_{3 \mathrm{PN}}, h_{\mathrm{NR}}\right)=0.976333$ and $\mathcal{O}\left(h_{3 \mathrm{PN}}, h_{\mathrm{NR}}\right)=0.973471$, respectively. Choosing functional forms for the cut-off function $\Theta$ compatible with the numerical data, but different from eq. 10$]$, the overlap integrals do not change significantly.

\section{B. Results}

In Table I we summarize the parameters of the binary configurations we consider, i.e. non-spinning $\mathrm{BH}-$ NS binaries with mass ratio $q=2, \operatorname{EOS} 2 \mathrm{H}, M_{\mathrm{NS}}=$ $(1.2,1.35) M_{\odot}$; we assume they are located at fixed luminosity distances $d_{\mathrm{L}}=100 / 500 / 1000 / 2000 \mathrm{Mpc}$, and identify the system with NS mass $\mathrm{M}_{\mathrm{NS}}$ at distance $d_{\mathrm{L}}$, with the label 2H_d $\mathrm{d}_{\mathrm{L}} \mathrm{M}_{\mathrm{NS}}$ (first column). In column 2 and 3 we show the NS compactness $\mathcal{C}$ and the rescaled tidal deformability $\tilde{\lambda}$ given by Eq. (8), respectively. Since, as discussed in Sec. III] we take into account the effect of cosmological redshift, the values of the redshift $z$ and of the redshifted cut-off frequencies $\bar{f}_{\text {cut }}=f_{\text {cut }} /(1+z)$ are also shown in Table If in the last column we show the signal-to noise ratio $\rho$, assuming the detector is ET.

\begin{tabular}{cccccc}
\hline \hline model & $\mathcal{C}$ & $\tilde{\lambda}\left(\mathrm{km}^{5}\right)$ & $z$ & $\bar{f}_{\text {cut }}(\mathrm{Hz})$ & $\rho$ \\
\hline 2H_100_120 & 0.117 & $7.31 \cdot 10^{4}$ & 0.023 & 779 & 563 \\
2H_500_120 & & 0.117 & 713 & 121 \\
2H_1000_120 & & 0.240 & 642 & 66 \\
2H_2000_120 & & 0.519 & 524 & 39 \\
\hline 2H_100_135 & 0.131 & $6.98 \cdot 10^{4}$ & 0.023 & 1061 & 619 \\
2H_500_135 & & 0.117 & 972 & 133 \\
2H_1000_135 & & 0.240 & 875 & 72 \\
2H_2000_135 & & 0.519 & 715 & 42 \\
\hline \hline
\end{tabular}

TABLE I. The NS compactness $\mathcal{C}$, rescaled tidal deformability $\tilde{\lambda}$, cosmological redshift, redshifted cut-off frequencies and signal-to noise ratio (assuming the detector is ET) are shown for the binary configurations considered in this section.

In Table II we show the errors obtained, applying the Fisher matrix approach, on the chirp mass $\mathcal{M}$, the symmetric mass ratio $\nu$, the rescaled tidal deformability $\tilde{\lambda}$, and the cut-off frequency $f_{\text {cut }}$, for the binary configurations shown in Table I. In the last column we show the error on the total mass of the system, $m$. The integrations on frequency (see Appendix A have been performed in the range $\left[10 \mathrm{~Hz}, 2 f_{\text {cut }}\right]$. Table II shows that the relative errors on the parameters increase of about a factor ten going from $100 \mathrm{Mpc}$ to $2 \mathrm{Gpc}$. It should be stressed that the analysis shows that $f_{\text {cut }}$ is uncorrelated with the other parameters. Therefore, for instance, the value of $\sigma_{\ln \tilde{\lambda}}$ is the same we would obtain using the waveform template given by Eq. (4) without $f_{\text {cut }}$.

The results obtained using the first strategy to estimate the NS compactness and the corresponding error, are presented in Table III where we show the relative percentage errors on: (i) the compactness $\mathcal{C}_{\text {cut }}$ derived

\begin{tabular}{cccccc}
\hline \hline model & $\sigma_{\ln \mathcal{M}}(\%)$ & $\sigma_{\ln \nu}(\%)$ & $\sigma_{\ln \tilde{\lambda}}(\%)$ & $\sigma_{\ln f_{\text {cut }}}(\%)$ & $\sigma_{\ln m}(\%)$ \\
\hline 2H_100_120 & $1.0 \cdot 10^{-4}$ & $2.0 \cdot 10^{-2}$ & 1.4 & 3.6 & $1.2 \cdot 10^{-2}$ \\
2H_500_120 & $5.7 \cdot 10^{-4}$ & $9.7 \cdot 10^{-2}$ & 6.0 & 14 & $5.8 \cdot 10^{-2}$ \\
2H_1000_120 & $1.3 \cdot 10^{-3}$ & $1.9 \cdot 10^{-1}$ & 10 & 22 & $1.2 \cdot 10^{-1}$ \\
2H_2000_120 & $3.2 \cdot 10^{-3}$ & $3.9 \cdot 10^{-1}$ & 15 & 27 & $2.3 \cdot 10^{-1}$ \\
\hline 2H_100_135 & $1.1 \cdot 10^{-4}$ & $1.7 \cdot 10^{-2}$ & 1.6 & 6.6 & $1.0 \cdot 10^{-2}$ \\
2H_500_135 & $6.1 \cdot 10^{-4}$ & $8.6 \cdot 10^{-2}$ & 6.7 & 26 & $5.1 \cdot 10^{-2}$ \\
2H_1000_135 & $1.4 \cdot 10^{-3}$ & $1.7 \cdot 10^{-1}$ & 11 & 40 & $1.0 \cdot 10^{-1}$ \\
2H_2000_135 & $3.4 \cdot 10^{-3}$ & $3.4 \cdot 10^{-1}$ & 17 & 49 & $2.0 \cdot 10^{-1}$ \\
\hline \hline
\end{tabular}

TABLE II. Percentage errors on the chirp mass, symmetric mass ratio, rescaled tidal deformability, cut-off frequency and total mass for the configurations listed in the first column.

from the frequency cut-off and the analytic fit (3), (ii) the compactness $\mathcal{C}_{\lambda}$ estimated by means of the universal relation (18), (iii) the weighted compactness $\mathcal{C}$ defined in Eq. (24). We do not show explicitly the value of the compactness obtained from this approach, because it coincides (with a discrepancy smaller than 1\%) with the "true" value shown in Table 【

\begin{tabular}{cccc}
\hline \hline model & $\sigma_{\ln \mathcal{C}_{\text {cut }}}(\%)$ & $\sigma_{\ln \mathcal{C}_{\lambda}}(\%)$ & $\sigma_{\ln \mathcal{C}}(\%)$ \\
\hline 2H_100_120 & 8.8 & 3.0 & 2.8 \\
2H_500_120 & 9.5 & 3.2 & 3.0 \\
2H_1000_120 & 10 & 3.5 & 3.3 \\
2H_2000_120 & 11 & 4.1 & 3.9 \\
\hline 2H_100_135 & 8.7 & 3.0 & 2.8 \\
2H_500_120 & 11 & 3.2 & 3.1 \\
2H_1000_120 & 14 & 3.6 & 3.5 \\
2H_2000_120 & 16 & 4.1 & 4.0 \\
\hline \hline
\end{tabular}

TABLE III. Relative percentage errors on the compactness $\mathcal{C}$ derived from the frequency cut-off fit (3), from the universal relation (18), and from the weighted mean 24.

From Table III we see that the relative percentage error in the NS compactness is of the order of $3 \%-4 \%$, with a very mild increase with the source distance. This weak dependence on $d_{\mathrm{L}}$ is due to the functional form of $\sigma_{\mathcal{C}_{\lambda}}$ which is dominated by the fit error $\sigma_{\text {fit }}$. Indeed, the latter is found to be always greater than the other two terms of Eq. 23) up to $d_{\mathrm{L}}=1 \mathrm{Gpc}$, when $\left(\partial \mathcal{C}_{\lambda} / \partial \ln \tilde{\lambda}\right)^{2} \sigma_{\ln \tilde{\lambda}}^{2}$ starts to be of the same order of magnitude of $\sigma_{\text {fit }}^{2}{ }^{3}$, The behavior of these quantities, as a function of the luminosity distance (for $M_{\mathrm{NS}}=1.2 M_{\odot}$ ), is shown in Fig. 2, We also see that the error $\sigma_{\mathcal{C}_{\lambda}}$ is reduced when $f_{\text {cut }}$ is included in the analysis, but this reduction is marginal. Thus, if our goal is to estimate the stellar compactness, the use of $f_{\text {cut }}$ in the data analysis does not introduce any significant improvement.

\footnotetext{
3 The error on the NS mass is always an order of magnitude smaller than the other two terms, for all the considered configurations.
} 




FIG. 2. We show the three terms appearing in Eq. (23), contributing to the relative error on $\sigma_{\mathcal{C}_{\lambda}}$, as functions of the luminosity distance $d_{\mathrm{L}}$, for $M_{\mathrm{NS}}=1.2 M_{\odot}$. The dot-dashed black curve represents the total error $\sigma_{\mathcal{C}_{\lambda}}^{2}$.

Let us now see whether using the second strategy is more convenient. The results are shown in Table IV. where we tabulate the relative percentage errors on the chirp mass $\mathcal{M}$, the symmetric mass ratio $\nu$, the rescaled tidal deformability and the compactness obtained from the fit (18). As in the previous case, we do not explicitly show the value of the compactness because it coincides with the value shown in Table II We can see that the

\begin{tabular}{ccccc}
\hline \hline model & $\sigma_{\ln \mathcal{M}}(\%)$ & $\sigma_{\ln \nu}(\%)$ & $\sigma_{\ln \tilde{\lambda}}(\%)$ & $\sigma_{\ln \mathcal{C}}(\%)$ \\
\hline 2H_100_120 & $1.0 \cdot 10^{-4}$ & $1.9 \cdot 10^{-2}$ & 1.3 & 3.0 \\
2H_500_120 & $5.6 \cdot 10^{-4}$ & $9.4 \cdot 10^{-2}$ & 5.3 & 3.1 \\
2H_1000_120 & $1.2 \cdot 10^{-3}$ & $1.8 \cdot 10^{-1}$ & 8.2 & 3.3 \\
2H_2000_120 & $3.1 \cdot 10^{-3}$ & $3.5 \cdot 10^{-1}$ & 10 & 3.6 \\
\hline 2H_100_135 & $1.1 \cdot 10^{-4}$ & $1.7 \cdot 10^{-2}$ & 1.5 & 3.0 \\
2H_500_135 & $6.0 \cdot 10^{-4}$ & $8.3 \cdot 10^{-2}$ & 6.1 & 3.2 \\
2H_1000_135 & $1.3 \cdot 10^{-3}$ & $1.6 \cdot 10^{-1}$ & 9.5 & 3.4 \\
2H_2000_135 & $3.3 \cdot 10^{-3}$ & $3.1 \cdot 10^{-1}$ & 12 & 3.6 \\
\hline \hline
\end{tabular}

TABLE IV. Relative percentage errors on the chirp mass, symmetric mass ratio, rescaled tidal deformability, and compactness computed the second data-analysis strategy proposed in this Section.

second strategy yields similar errors for the NS compactness, of the order of $3 \%-4 \%$. Conversely, if we compare the relative percentage error on $\tilde{\lambda}$ computed with this strategy (column 4 of Table IV), with the same given in column 4 of Table III we see that the error is reduced when the second strategy is applied, and the reduction is up to $\sim 30 \%$ for more distant sources. It may also be noted that, unlike $\mathcal{C}, \sigma_{\tilde{\lambda}}$ varies from $\sim 1 \%$ to $\sim 10 \%$.

At this point we may ask whether $\mathcal{C}$ is the best parameter to be used to gain information on the NS equation of state. This problem will be discussed in detail in the next Section.

\section{CHOOSING THE MOST SUITABLE QUANTITY TO CONSTRAIN THE NS EOS}

In this section we compare the relative error on the two parameters which allow to constrain the equation of state of matter in the NS interior, i.e. $\lambda$ (or equivalently $\tilde{\lambda}$ ) and $\mathcal{C}$. Tidal effects in NS-NS coalescing binaries may be revealed by second generation detectors AdvLIGO/Virgo within a distance of $100 \mathrm{Mpc}$, provided the signal-to noise ratio is larger than 16 12]; therefore,we extend the analysis of the previous section, based on the Fisher matrix approach, to NS-NS and BH-NS configurations, choosing a larger set of EoS to model the NS interior, and considering the sensitivity curves of both AdvLIGO/Virgo and of the Einstein Telescope. We do not include $f_{\text {cut }}$ in this analysis, because its role has already been discussed in Section IVB. We use 7 different EoS, which are described in terms of piecewise polytropes [41, with three pieces for the NS inner core and one piece for the crust. We select: (i) two EoS derived using variational-method approaches, APR4 and WFF1 44,,45]; (ii) two based on the relativistic Brueckner-Hartree-Fock approach, MPA1 and ENG 46, 47]; (iii) one based on the potential-method SLy4 [48]; (iv) one derived within the relativistic meanfield approach MS1 [49]; (v) one relativistic mean-field theory EoS which includes hyperons H4 [50]. It is useful to remind that stiffer EoS correspond to more deformable stars, larger values of $\tilde{\lambda}$ and smaller values of $\mathcal{C}$. Ordering our EoS from the softest to the stiffest, we find WFF1, APR4, SLy4, ENG, MPA1, H4, MS1.

We consider NS-NS binaries with mass ratio $q=1$, and BH-NS binaries with mass ratio $q=2,4$; in the first case both stars are modeled with the same equation of state. The error on the tidal deformability is directly computed by means of a $5 \times 5$ Fisher Matrix for the set of parameters $\boldsymbol{\theta}=\left(t_{c}, \phi_{c}, \ln \mathcal{M}, \ln \nu, \tilde{\lambda}\right)$. The uncertainty on $\mathcal{C}$ is obtained in terms of the uncertainty on $\tilde{\lambda}$, using the fit (18) (see Eq. (23)). For each detector we consider prototype binaries at fixed distances:

- For the Advanced detectors, we analyze systems at luminosity distance $d_{\mathrm{L}}=(20,100) \mathrm{Mpc}$. In this case the $S_{h}(f)$ is taken to be the ZERO_DET_high $\mathrm{P}$ anticipated sensitivity curve [51].

- For ET we consider binaries at distance up to $d_{\mathrm{L}}=$ $1 \mathrm{Gpc}$, and use the noise spectral density given by the analytic fit (17).

We employ the standard PN template

$$
h_{\mathrm{PN}}=\mathcal{A}_{3 \mathrm{PN}}(f) e^{i\left(\psi_{\mathrm{PP}}+\psi_{\mathrm{T}}\right)} .
$$

All quantities are suitably redshifted. The integrations are performed in the frequency range $\left[f_{\mathrm{min}}, f_{\mathrm{ISCO}}\right]$, where $f_{\min }$ is set to $20 \mathrm{~Hz}$ for AdvLIGO/Virgo and $10 \mathrm{~Hz}$ for $\mathrm{ET}$, and $f_{\mathrm{ISCO}}=\left(6^{3 / 2} m\right)^{-1}$. The results are shown in Figs. 3 and 4 . where we plot the intervals $\tilde{\lambda} \pm \sigma_{\tilde{\lambda}}$ (left panels of Fig. 3, and Fig. 4) and $\mathcal{C} \pm \sigma_{\mathcal{C}}$ (right panels of 
Fig. 33 as functions of the NS mass for AdvLIGO/Virgo and ET, respectively. In the headline of each panel we indicate the detector, the mass ratio and the source distance.

Let us start discussing the results for AdvLIGO/Virgo shown in Fig. 3 In the upper, left panel, we consider NS-NS binaries as close as $20 \mathrm{Mpc}$; we see that, for the softer EoS (WFF1, APR4, SLy4, ENG, MPA1) the tidal deformability $\tilde{\lambda}$ is weakly dependent on the NS mass; for stiffer EoS (H4, MS1) $\tilde{\lambda}$ exhibits a more pronounced dependence on $M_{\mathrm{NS}}$; in addition, for each $\operatorname{EoS} \tilde{\lambda}$ varies in ranges ( $1 \sigma$ intervals) which are more separated for stiffer EoS, and for masses smaller that $\sim 1.8 M_{\odot}$. Conversely, the compactness $\mathcal{C}$ shown in the right, upper panel does not seem to be a good indicator of the EoS.

If the sources are farther away, say at $100 \mathrm{Mpc}$ as shown in the middle panels, chances to discriminate among the EoS decrease, because the $1 \sigma$ intervals become larger, and only when the EoS is stiff and the mass is lower than $\sim 1.5 M_{\odot}$, the quantity $\tilde{\lambda}$ can be used as EoS indicator. In the four lower panels of Fig. 3 we consider BH-NS binaries with $q=2$ and $d_{\mathrm{L}}=20$ and $d_{\mathrm{L}}=100 \mathrm{Mpc}$, respectively. We see that advanced detectors will be able to extract some information on the EoS from $\tilde{\lambda}$ only if the source is very close and the NS mass is lower than $\sim 1.5 M_{\odot}$, but it should be reminded that the rate of $\mathrm{BH}-\mathrm{NS}$ coalescence is much smaller than that of NS-NS, therefore to obtain a reasonable detection rate one should have access to a much larger volume space, as that allowed by the third generation detectors like ET. When $q>2$, the chances to discriminate among the EoS are even smaller. In any event, comparing the right and the left panels of Fig. 3 we can conclude that $\tilde{\lambda}$ is the parameter to use if we want to discriminate among different EoS, although its effectiveness decreases with the source distance. Since this is true also for third generation detectors, in the following we shall consider only the plots for $\tilde{\lambda}$. They are shown in Fig. 4 for the detector ET. As noted before, the possibility to discriminate between different EoS decreases as the distance increases; however, if the source is a NS-NS system (upper panels) it remains acceptable even when $d_{\mathrm{L}}$ is as large as the ET horizon distance, which is estimated to be about $2 \mathrm{Gpc}$ (right panel). If the source is close (left panel $d_{\mathrm{L}}=100$ $\mathrm{Mpc}$ ), the error on $\tilde{\lambda}$ is very small and, unless the NS masses are close to the maximum observed mass there are good chances to identify at least the class to which the EoS belongs. The remaining four panels of Fig. 4 refer to BH-NS systems at a distance of $100 \mathrm{Mpc}$ (left) and of 2 Gpc (right). The middle panels refer to systems with mass ratio $q=2$, the lower panels to more realistic systems with $q=4$. We see that there is no way to give reliable information on the EoS if this kind of source is at cosmological distance, regardless of the value of $q$. For closer systems, and if the mass is smaller than a certain value, we could infer if the EoS is soft or stiff; for instance this could be possible for sources at $d_{\mathrm{L}}=100 \mathrm{Mpc}$ if $M_{\mathrm{NS}}$ is smaller than $\sim 1.7 M_{\odot}$, for systems with $q=2$, and if
$M_{\mathrm{NS}}$ is smaller than $\sim 1.5 M_{\odot}$, for systems with $q=4$.

\section{CONCLUDING REMARKS}

In this work we have discussed how to extract information on the EoS of matter in a neutron star interior, using the gravitational wave signal emitted in NS-NS or $\mathrm{BH}-\mathrm{NS}$ coalescence. In a few years the second generation of interferometric detectors AdvLIGO/Virgo should detect GW signals emitted by these sources, and chances of detection will significantly increase with the third generation detectors. The information on the NS EoS is encoded in the signal emitted during the latest phases of inspiralling before merging by means of two quantities: the NS tidal deformability and, when tidal disruption occurs before merger, the cut-off frequency. Assuming that a signal emitted by one or more of such systems is detected, we have evaluated the accuracy with which different parameters can be determined, using different data analysis strategies based on the Fisher matrix approach and on analytical fits of the relevant quantities obtained by numerical simulations of the coalescence process.

Our main results can be summarized as follows.

- Using $f_{\text {cut }}$ to gain information on the NS EoS.

We find that, using $f_{\text {cut }}$ as free parameter in the Fisher matrix approach and the first proposed strategy, the NS compactness $\mathcal{C}$ can be estimated with a relative error of the order of $3 \%-4 \%$, and that its estimate has a weak dependence on $d_{\mathrm{L}}$, being the error dominated by the error on the fit $\mathcal{C}(\lambda)$ (see Eq. 23p). The situation does not change if we use the second strategy, i.e. if we do not include $f_{\text {cut }}$ as unknown parameter in the Fisher matrix, and express the waveform template only in terms of the tidal deformation, using the fits $f_{\text {cut }}(\mathcal{C})$ and $\mathcal{C}(\lambda)$. Thus, if our goal is to estimate $\mathcal{C}$, the use of $f_{\text {cut }}$ in the analysis is ineffective. However, if the goal is to estimate the tidal deformability $\tilde{\lambda}$, it is better to use the second strategy because the error reduces up to $30 \%$ for more distant sources. To make this analysis we have used the same models used in 8 , to obtain the fit $f_{\text {cut }}(\mathcal{C})$, i.e. non spinning BH-NS binaries with mass ratio $q=2$, with the neutron star modeled using a set of piecewise polytropes.

This study could, and should, be extended to more general BH-NS binaries, with different values of the mass ratio and different EoS, and including the $\mathrm{BH}$ spin. The extension to spinning BHs is important because when the mass ratio is larger, or the EoS is less deformable, NS tidal disruption can occur only if the $\mathrm{BH}$ is rapidly rotating. Such extension will be possible only when fully relativistic, numerical simulations will extend the domain of validity of the fit $f_{\text {cut }}(\mathcal{C})$. In addition, it should be stressed that the presence of a frequency cutoff in the GW 

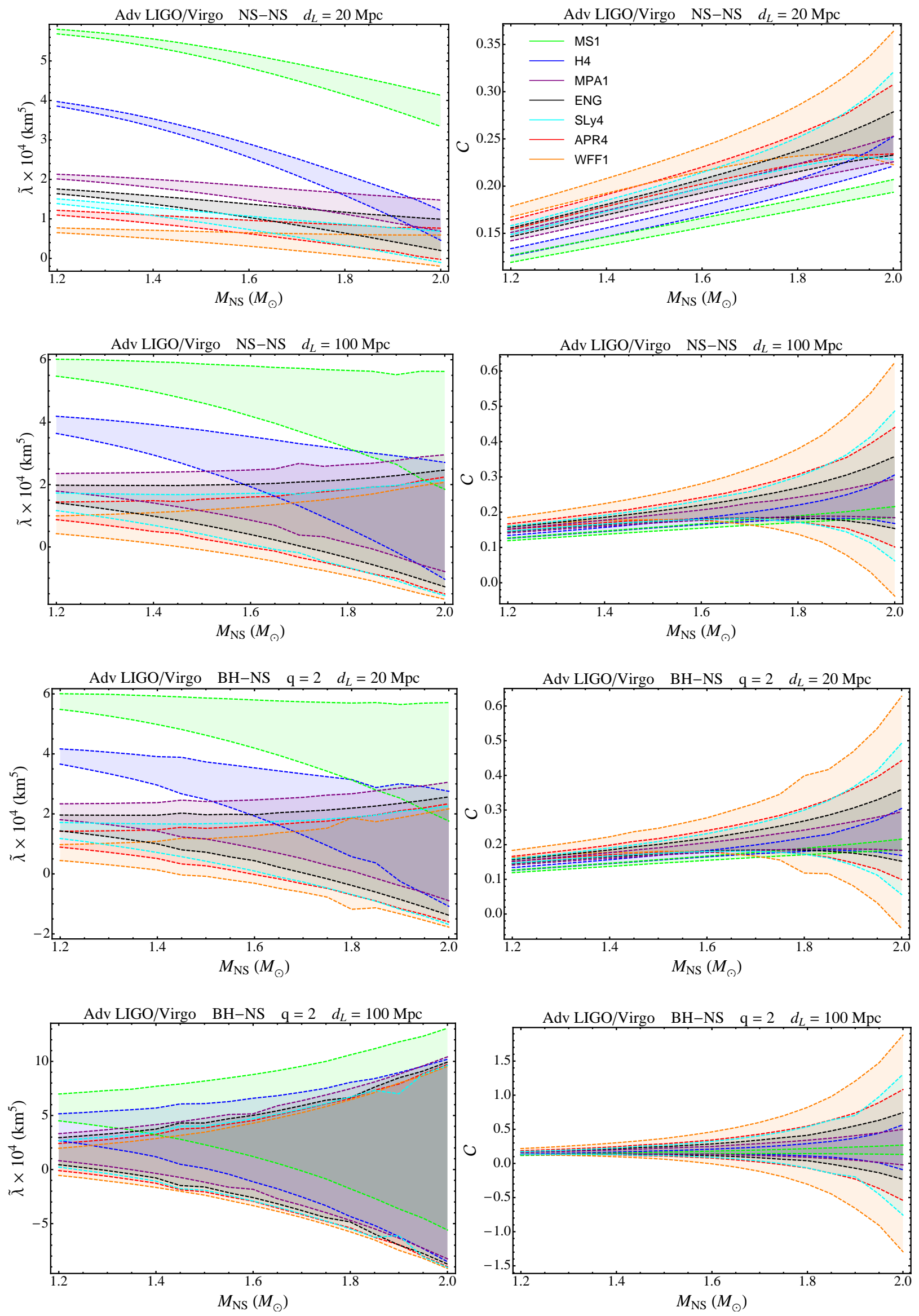

FIG. 3. The quantities $\tilde{\lambda} \pm \sigma_{\tilde{\lambda}}$ (left column) and $\mathcal{C}_{\lambda} \pm \sigma_{\mathcal{C}_{\lambda}}$ (right column) are plotted as functions of the NS mass, at different luminosity distances $d_{\mathrm{L}}$, for NS-NS binaries and for BH-NS systems with mass ratio $q=2$. The parameter errors $\sigma_{\tilde{\lambda}}$ and $\sigma_{\mathcal{C}_{\lambda}}$ are evaluated for AdvLIGO/Virgo. Different bands correspond to different NS EoS. 

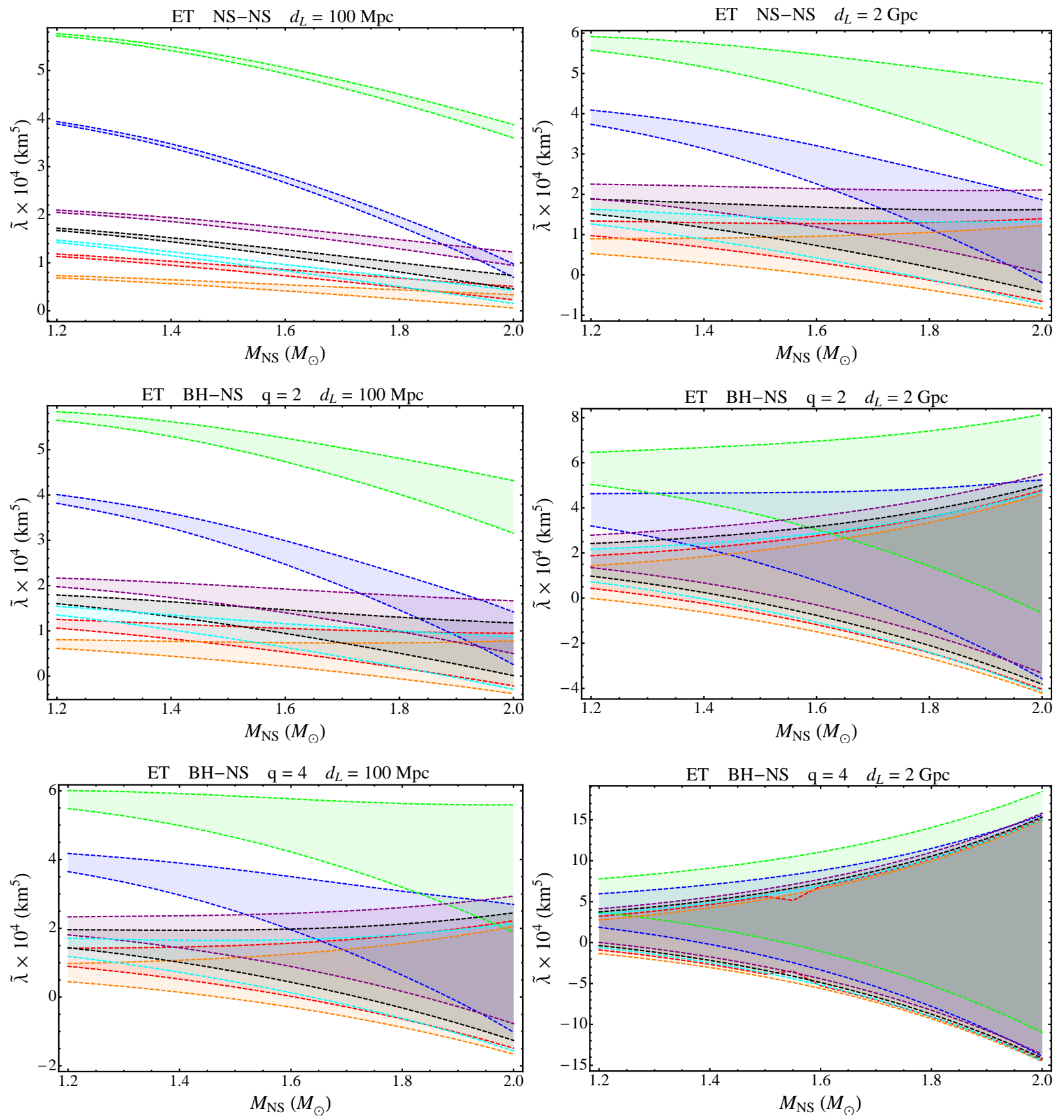

FIG. 4. The quantity $\tilde{\lambda} \pm \sigma_{\tilde{\lambda}}$ is plotted as a function of the NS mass, for sources at luminosity distance $d_{\mathrm{L}}=100 \mathrm{Mpc}$ (left panels) and $d_{\mathrm{L}}=100 \mathrm{Gpc}$ (right panels), for NS-NS systems and for BH-NS systems with $q=2$ and $q=4$, and for the same EoS considered in Fig. 3. The errors are evaluated for the Einstein Telescope. 
signal indicates that the NS has been tidally disrupted before merging with the companion; if the torus of dense matter which subsequently forms has sufficiently high mass, a Short Gamma-Ray Burst could be powered. Therefore, estimating $f_{\text {cut }}$ as accurately as possible is also important.

- Comparing $\tilde{\lambda}$ and $\mathcal{C}$ as EoS indicators.

We have discussed and compared the effectiveness of $\tilde{\lambda}$ and $\mathcal{C}$ in discriminating between different EoS. To this aim, we have considered NS-NS and NS$\mathrm{BH}$ binaries, modeled using a large set of EoS, to be detected by $2^{\text {nd }}$ and $3^{\text {rd }}$ generation interferometers. We find that $\tilde{\lambda}$ is much better than $\mathcal{C}$ to constrain the NS equation of state.

For sources at distance $d_{\mathrm{L}} \lesssim 100 \mathrm{Mpc}$, a signal emitted by NS-NS binaries detected by Advanced LIGO/Virgo would allow to discriminate between different EoS, if $M_{\mathrm{NS}} \lesssim 1.5 M_{\odot}$. If the signal is detected by ET, different EoS will be discerned even for larger distances and larger NS masses. Conversely, it is very unlikely that BH-NS binaries will allow us to discriminate between different EoS: this could happen only in the unlikely case of a signal detected by ET from a system with $M_{\mathrm{NS}} \lesssim 1.3 M_{\odot}$ and $q \lesssim 2$.

Other improvement of this strategy that should be pursued are:

- Use of more realistic gravitational wave templates, aimed at reproducing in detail the full merger of BH-NS binaries [52].

- Correlation of other binary parameters related to the neutron star structure. For instance, it has recently been shown that the peak frequency of the post merger GW signal from NS-NS mergers is strongly related with the NS radius [53.

- Use of Bayesian inference for GW parameter estimation based on Markov Chain Monte Carlo algorithms [24, 54.

\section{Appendix A: Parameter estimation: basic Theory}

In this section we briefly recall the key points of parameter estimation theory. Given a signal of the form $h(t, \boldsymbol{\theta})$, we want to extract the physical parameters $\boldsymbol{\theta}$ and estimate the errors $\Delta \boldsymbol{\theta}=\boldsymbol{\theta}-\hat{\boldsymbol{\theta}}$, were we assume $\hat{\boldsymbol{\theta}}$ to be the true values of the parameters. To this aim we have to compute $p(\boldsymbol{\theta} \mid s)$, namely the probability of measuring the value $\boldsymbol{\theta}$, given the detector output $s(t)$

$$
s(t)=h(t, \boldsymbol{\theta})+n(t),
$$

where $n(t)$ represents the detector noise, which is assumed to be stationary. It has been shown that [23]

$$
p(\boldsymbol{\theta} \mid s) \propto p^{(0)}(\boldsymbol{\theta}) e^{\left.-\frac{1}{2}(h(\boldsymbol{\theta})-s \mid h(\boldsymbol{\theta})-s)\right)},
$$

where $p^{(0)}(\boldsymbol{\theta})$ is the prior probability on the physical set of parameters and the constant of normalization is independent of $\boldsymbol{\theta}$. We define the inner product $(\cdot \mid \cdot)$ as

$$
(g \mid h)=2 \int_{-\infty}^{\infty} \frac{\tilde{h}(f) \tilde{g}^{\star}(f)+\tilde{h}^{\star}(f) \tilde{g}(f)}{S_{h}(f)} d f
$$

where $\tilde{h}(f)$ is the Fourier transform of $h(t)$ and * denotes complex conjugation. The integration range can be different from $[-\infty,+\infty]$, if specified. $S_{n}(f)$ is the noise spectral density (PSD) of the detector considered. For a given measurement the values of the source parameters can be estimated as those which maximize the probability distribution (A2): this is the so called maximumlikelihood estimator. Moreover, we define the signal-tonoise ratio $\rho$, such that

$$
\rho^{2}=(h \mid h)=4 \int_{0}^{\infty} \frac{|\tilde{h}(f)|^{2}}{S_{h}(f)} d f
$$

evaluated at $\boldsymbol{\theta}=\hat{\boldsymbol{\theta}}$. In the following we will consider the limit of large signal to noise ratio (SNR), in which $p(\boldsymbol{\theta} \mid h)$ is sharply peaked around the true values of the source parameters. It can be easily proved that in this limit

$$
p(\boldsymbol{\theta} \mid s) \propto p^{(0)}(\boldsymbol{\theta}) e^{-\frac{1}{2} \Gamma_{a b} \Delta \theta^{a} \Delta \theta^{b}},
$$

where

$$
\Gamma_{a b}=\left(h_{, a} \mid h_{, b}\right)
$$

evaluated at $\boldsymbol{\theta}=\hat{\boldsymbol{\theta}}$, is the Fisher information matrix [39]. This allows to define the variance-covariance matrix $\Sigma^{a b}$ as 4

$$
\Sigma^{a b}=\left\langle\Delta \theta^{a} \Delta \theta^{b}\right\rangle=\left(\boldsymbol{\Gamma}^{-1}\right)^{a b},
$$

where $\boldsymbol{\Gamma}^{-1}$ is the inverse of Fisher matrix. In this way we can define the error associated to the parameter $\theta^{a}$ as

$$
\sigma_{a}=\left\langle\left(\Delta \theta^{a}\right)^{2}\right\rangle^{1 / 2}=\sqrt{\Sigma^{a a}},
$$

and the correlation coefficient between $\theta^{a}$ and $\theta^{b}$ as

$$
c_{a b}=\frac{\left\langle\Delta \theta^{a} \Delta \theta^{b}\right\rangle}{\Sigma^{a a} \Sigma^{b b}}=\frac{\Sigma^{a b}}{\sqrt{\Sigma^{a a} \Sigma^{b b}}},
$$

with $c_{a b} \in[-1,1]$. Eq. A5 holds whether $p^{(0)}(\boldsymbol{\theta})$ is uniform around $\hat{\boldsymbol{\theta}}$ or not. In the first case, the probability distribution for the parameters takes a Gaussian form. Otherwise $p(\boldsymbol{\theta} \mid s)$ doesn't represent the maximumlikelihood estimate, and it may not be Gaussian. In the special case when $p^{(0)}$ is Gaussian, namely

$$
p^{(0)}(\boldsymbol{\theta}) \propto e^{-\frac{1}{2} \Gamma_{a b}^{(0)}\left(\theta^{a}-\bar{\theta}^{b}\right)\left(\theta^{b}-\bar{\theta}^{b}\right)}
$$

\footnotetext{
4 Angular brackets denote average on the probability distribution defined by Eq. A5.
} 
the probability distribution $p(\boldsymbol{\theta} \mid s)$ is Gaussian with covariance matrix given by

$$
\boldsymbol{\Sigma}=\left(\boldsymbol{\Gamma}+\boldsymbol{\Gamma}^{(0)}\right)^{-1}
$$

We note that in general $p(\boldsymbol{\theta} \mid s)$ is peaked around $\langle\boldsymbol{\theta}\rangle$, which is, in general, different from $\overline{\boldsymbol{\theta}}$ and $\hat{\boldsymbol{\theta}}$.

\section{ACKNOWLEDGMENTS}

We would like to thank M. Fortin and L. Pagano for useful discussions. A.M. is supported by a "Virgo EGO Scientific Forum" (VESF) grant.
[1] http://www.ligo.caltech.edu http://www.ego-gw.it

[2] http://www.et-gw.eu/

[3] T. Hinderer, Astrophys. J. 677, 1216 (2008); ibid., 697, 964 (2009).

[4] E.E. Flanagan, T. Hinderer, Phys. Rev. D77, 021502 (2008).

[5] M. Vallisneri, Phys. Rev. Lett. 84, 3519 (2000).

[6] V. Ferrari, L. Gualtieri and F. Pannarale, Phys. Rev. D 81, 064026 (2010).

[7] T. Hinderer, B. D. Lackey, R. N. Lang and J. S. Read, Phys. Rev. D 81, 123016 (2010).

[8] K. Kyutoku, M. Shibata, K. Taniguchi, Phys. Rev. D. 82, 044049 (2010).

[9] M. D. Duez, F. Foucart, L. E. Kidder, C. D. Ott and S. A. Teukolsky, Class. Quant. Grav. 27, 114106 (2010).

[10] F. Pannarale, L. Rezzolla, F. Ohme and J. S. Read, Phys. Rev. D. 84, 104017 (2011).

[11] B. D. Lackey, K. Kyutoku, M. Shibata, P.R. Brady, J.L. Friedman, Phys. Rev. D 85044061 (2012).

[12] T. Damour, A. Nagar, L. Villain, Phys. Rev. D 85, 123007 (2012).

[13] J. S. Read, L. Baiotti, J. D. E. Creighton, J. L. Friedman, B. Giacomazzo, K. Kyutoku, C. Markakis and L. Rezzolla et al., Phys. Rev. D 88, 044042 (2013).

[14] A. Maselli, V. Cardoso, V. Ferrari, L. Gualtieri and P. Pani, Phys. Rev. D 88, 023007 (2013).

[15] R. Narayan, B. Paczynski and T. Piran, Astrophys. J. 395, L83 (1992).

[16] I. Bartos, P. Brady and S. Marka, Class. Quant. Grav. 30, 123001 (2013).

[17] J. M. Lattimer and M. Prakash, Phys. Rept. 442, 109 (2007).

[18] K. Yagi and N. Yunes, Science 341, 365 (2013); Phys. Rev. D 88, 023009 (2013).

[19] T. Damour and A. Nagar, Phys. Rev. D 80, 084035 (2009).

[20] T. Binnington and E. Poisson, Phys. Rev. D 80, 084018 (2009).

[21] J. Vines, E.E. Flanagan, T. Hinderer, Phys. Rev. D 83, 084051 (2011).

[22] D. Bini, T. Damour, G. Faye, Phys. Rev. D 85124034 , (2012).

[23] C. Cutler and E. E. Flanagan, Phys. Rev. D 49, 2658 (1994).

[24] R. Balasubramanian, B. S. Sathyaprakash and S. V. Dhurandhar, Phys. Rev. D 53, 3033 (1996).

[25] W. Del Pozzo, T. G. F. Li, M. Agathos, C. V. D. Broeck and S. Vitale, Phys. Rev. Lett. 111, 071101 (2013).
[26] M. Shibata, K. Kyutoku, T. Yamamoto and K. Taniguchi, Phys. Rev. D 79, 044030 (2009); 85, 127502(E) (2012).

[27] K. Kyutoku, H. Okawa, M. Shibata and K. Taniguchi, Phys. Rev. D 84, 064018 (2011).

[28] R. Narayan, New J. Phys. 7, 199 (2005).

[29] L. Baiotti, T. Damour, B. Giacomazzo, A. Nagar and L. Rezzolla, Phys. Rev. Lett. 105, 261101 (2010); Phys. Rev. D 84, 024017 (2011).

[30] S. Bernuzzi, M. Thierfelder and B. Bruegmann, Phys. Rev. D 85, 104030 (2012).

[31] S. Bernuzzi, A. Nagar, M. Thierfelder and B. Bruegmann, Phys. Rev. D 86, 044030 (2012).

[32] K. Hotokezaka, K. Kyutoku and M. Shibata, Phys. Rev. D 87, 044001 (2013).

[33] D. Radice, L. Rezzolla and F. Galeazzi, Mon. Not. Roy. Astron. Soc. published online, doi:10.1093/mnrasl/slt137 (2013).

[34] T. Damour, B. R. Iyer and B.S. Sathyaprakash, Phys. Rv. D 62, 084036 (2000).

[35] T. Damour, A .Nagar, M. Trias, Phys. Rev. D 83, 024006 (2011).

[36] L. Blanchet, living Rev. Relativity 9, 4 (2006).

[37] C. Messenger and J. Read, Phys. Rev. Lett. 108, 091101 (2012).

[38] Planck Collaboration, arXiv:1303:5062 (2013).

[39] E. Poisson and C. M. Will, Phys. Rev. D 52, 2 (1995).

[40] B.S. Sathyaprakash and B.F. Schultz, Living Reviews in Relativity 12, 2 (2009).

[41] J. Read et al., Phys. Rec. D 79, 124033 (2009).

[42] J. Vines, E. Flanagan, gr-qc: 1009.4919 (2010).

[43] L. Lindblom, B.J. Owen and D.A. Brown, Phys. Rev. D 78, 124020 (2008).

[44] A. Akmal, V.R. Pandharipande, and D.G. Ravenhall, Phys. Rev. C 58, 1804 (1998).

[45] R.B. Wiringa, V. Fiks, and A. Fabrocini, Phys. Rev. C 38, 1010 (1988).

[46] H. Muther, M. Prakash, and T.L. Ainsworth, Phys. Lett. B (199), 469 (1987).

[47] L. Engvik et al., Astrophys. J. 469, 794 (1996).

[48] F. Douchin and P. Haensel, Astron. Astrophys. 380, 151 (2001).

[49] H. Muller and B.D. Serot, Nucl. Phys. A606, 508 (1996).

[50] B.D. Lackey, M. Nayyar, and B.J. Owen, Phys. Rev. D 73, 024021 (2006).

[51] D. Shoemaker, https://dcc.ligo.org/cgi-bin/ DocDB/ShowDocument?docid=2974.

[52] F. Pannarale,E. Berti, K. Kyutoku and M. Shibata, Phys. Rev. D 88, 084011 (2013). 
[53] A. Bauswein, H.T. Janka, K.Hebeler and A. Schwenk, Phys. Rev. D 86, 063001 (2012).

[54] A. Bauswein and H. -T. .Janka, Phys. Rev. Lett. 108, 011101 (2012). 